\title{
Eficacia de la entrevista motivacional para promover la adherencia terapéutica en pacientes con Diabetes Mellitus tipo ${ }^{*}$
}

Efficacy of Motivational Interviewing to Promote Therapeutic Adherence in Diabetes Mellitus Type 2 Patients

Recibido: 1 de noviembre de 2013 | Revisado: 19 de octubre de 2014 | Aceptado: 9 de diciembre de 2014

\author{
Ismael García Cedillo ** \\ Brenda Verónica MORAles AntúneZ *** \\ Universidad Autónoma de San Luis Potosí, México
}

doi:10.11144/Javeriana.upsy14-2.eemp

Para citar este artículo: García, I., \& Morales, B. V. (2015). Eficacia de la entrevista motivacional para promover la adherencia terapéutica en pacientes con Diabetes Mellitus tipo 2. Universitas Psychologica, 14(2), 511-522. http://dx.doi.org.10.11144/ Javeriana.upsy14-2.eemp

* La investigación contó con el apoyo de la Secretaría de Salud de San Luis Potosí y de la Universidad Autónoma de San Luis Potosí.

** Profesor-investigador del Instituto de Investigación y Posgrado de la Universidad Autónoma de San Luis Potosí, México. Miembro del Sistema Nacional de Investigadores. Correo electrónico: ismaelgace@ yahoo.com.mx

**** Egresada de la Maestría en Psicología de la Universidad Autónoma de San Luis Potosí, México. Psicóloga de una Unidad de Servicios de Apoyo a la Educación Regular de la Secretaría de Educación Estatal de San Luis Potosí, México. Correo electrónico: brenda_sony@hotmail.com

\section{RESUMEN}

Se reporta un estudio pre-post con dos grupos, control y experimental, en el que se implementó una intervención individual con el enfoque de la entrevista motivacional (EM) para aumentar la adherencia terapéutica (AT) de pacientes con Diabetes Mellitus tipo 2 (DM2). Participaron 11 pacientes en grupo control y 11 en el experimental, de una institución de salud en México; se aplicaron dos instrumentos para evaluar su AT y estilo de vida. La intervención consistió en seis sesiones individuales de 40 minutos, basadas en la EM. Siete de los once pacientes del grupo experimental cambiaron de nivel de AT. Los niveles de glucosa del grupo experimental mostraron diferencias significativas $(p \leq 0.05)$. Se concluye que la EM resultó efectiva para aumentar la AT en pacientes con DM2 y que las intervenciones diseñadas para promover la AT deben considerar que, por cuestiones personales y culturales, a las mujeres les resulta más difícil.

Palabras clave

Diabetes Mellitus tipo 2; adherencia terapéutica; entrevista motivacional

\section{A B S T R A C T}

This paper reports pre-post test results on two groups; the purpose is to enhance therapeutic adherence (TA) of Diabetes Mellitus Type 2 (DM2) patients. The sample included 11 patients in the control group and 11 in the experimental group, being served at a public hospital in México. All patients were administered two tests in order to assess their lifestyle and therapeutic adherence. Also pre and post intervention glucose levels were obtained. The intervention consisted of six 40-minutes individual meetings based on motivational interviewing. Seven out of the eleven patients in the intervention group changed their TA level. The pre and post intergroup glucose levels of the experimental group showed statistical differences $(\mathrm{p} \leq$ 0.05). We concluded the intervention was effective to increase the TA in DM2 patients. However, it is important to understand that women, due to personal and cultural considerations, face more difficulties to reach a good level of AT.

Keywords

Diabetes Mellitus Type 2; therapeutic adherence; motivational interviewing 


\section{Introducción}

En el mundo hay más de 371 millones de personas con Diabetes Mellitus tipo 2 (DM2); se calcula que en el año 2030 la prevalencia alcance a 552 millones de personas (International Diabetes Federation, 2013). La Diabetes Mellitus constituye una enfermedad de alto costo tanto para los sistemas de salud como para los pacientes. Estos últimos solicitan con mayor frecuencia atención en las unidades de salud, necesitan más medicamentos, tienen una mayor probabilidad de ingresar a los servicios de urgencias y requieren hospitalizaciones más prolongadas, debido a las múltiples complicaciones (Rodríguez, Reynales, Jiménez, Juárez, \& Hernández, 2010). Cerca del $80 \%$ de las muertes por diabetes se registran en países de ingresos bajos y medios. Casi la mitad de esas muertes corresponden a personas de menos de 70 años, y un $55 \%$ a mujeres (Organización Panamericana de la Salud [OPS], 2011; Organización Mundial de la Salud [OMS], 2012). El $90 \%$ de las personas que padecen diabetes presenta el tipo 2 de la enfermedad.

México presenta una transición epidemiológica caracterizada por un predominio cada vez mayor de enfermedades no transmisibles y prevenibles, relacionadas con el estilo de vida, entre las principales se encuentran el sobrepeso y obesidad, la hipertensión y la DM2; esta última se ha convertido en la primera causa de muerte en personas de 20 a 65 años, y la segunda, si se consideran todas las edades (Secretaría de Salud, 2013). De acuerdo con el Gobierno de la República (2013), la DM2 tiene una prevalencia de $9.2 \%$ del total de la población (8.6 de los hombres y 9.67 de las mujeres, de acuerdo con Hernández-Ávila, Gutiérrez, \& Reynoso-Noverón, 2013), y constituye, sin duda alguna, el mayor reto que enfrenta el Sistema Nacional de Salud; ya desde 2007 se señalaba que "es la principal causa de demanda de atención médica en consulta externa, una de las principales causas de hospitalización, y la enfermedad que consume el mayor porcentaje del gasto de las instituciones públicas" (Secretaría de Salud, 2007, p. 29). Del total de pacientes diabéticos, el $46.95 \%$ reportó también diagnóstico de hipertensión y $4.47 \%$ antecedentes de infarto del corazón (Hernández-Ávila et al., 2013). La Encuesta Nacional de Salud 2012 reporta que un poco más del $80 \%$ de las personas con diagnóstico médico previo de diabetes recibe tratamiento médico para su control (Instituto Nacional de Salud Pública [INSP], 2012).

Según la International Diabetes Federation (2013), México ocupa el sexto lugar de personas con diabetes en el mundo. La incidencia de casos nuevos es de 442.23 por cada 100 mil entre las mujeres y de 326.81 casos por cada 100 mil entre los hombres (Instituto Nacional de Estadística y Geografía [INEGI], 2013). El costo del tratamiento en México, de acuerdo con diversos estudios, oscila entre 700 y 3200 dólares anuales por paciente, Las estimaciones del gasto para el sistema de salud son muy variables, van del 5 al $14 \%$ del gasto total (Hernández-Ávila et al., 2013; Hernández-Romieu, Elnecavé-Olaiz, Huerta-Uribe, \& Reynoso-Noverón, 2011).

A pesar de que la diabetes puede ser diagnosticada fácilmente y de que existen cada vez más tratamientos disponibles para ayudar a las personas con esta enfermedad a mantener bajo control sus niveles de glucosa, las consecuencias del mal control y la mortalidad continúan en aumento.

El tratamiento intensivo y adecuado de la DM2 se relaciona con el retardo en la aparición y progresión de las complicaciones crónicas de la enfermedad. Este tratamiento debe ser integral, con el objetivo de alcanzar un alto grado de adherencia terapéutica (AT) y así mantener niveles fisiológicos óptimos de glucemia, lo que permitirá prevenir, retardar o minimizar las complicaciones tardías de la enfermedad (Leytur, 2002). Sin embargo, lograr la AT está lejos de ser fácil (Durán, Rivera, \& Franco, 2001). En los países desarrollados, por ejemplo, la AT es del $50 \%$, comparado con el $25 \%$ en los países con menor desarrollo (OMS, 2004); por ello, es necesario modificar las estrategias de control de esta enfermedad para incluir la educación en diabetes y su tratamiento, el cual comprende la realización de actividad física, la alimentación de acuerdo a las características individuales del enfermo, el control de peso y el autocuidado de la salud, así como la 
toma de medicamentos de la manera en que fue prescrita (Secretaría de Salud, 2007).

La AT implica que el paciente realice cambios en su estilo de vida que, por ser muy difíciles de lograr, con frecuencia necesitan una intervención psicológica para ayudar a implementarlos. Como la DM2 constituye una enfermedad crónico-degenerativa, no alcanzar una alta AT implica para el paciente el deterioro de su capacidad funcional y de su sensación de bienestar (Mena, Martín, Simal, Bellido, \& Carretero, 2006). Al respecto, Balkrishnan et al. (2003) reportaron que en pacientes mayores de 65 años del sur de los EE. UU. una baja AT a los medicamentos estuvo asociada con un incremento en la utilización de los servicios de salud. En distintos estudios metaanalíticos se encontró lo siguiente: Vermeire et al. (2005) señalan que la baja AT en diabetes se asocia a serias complicaciones vasculares, nefrológicas, neurológicas y oftalmológicas. DiMatteo, Lepper y Croghan (2000) encontraron que la baja AT se relaciona significativamente con la depresión, pero no con la ansiedad. Cramer (2004) reportó que la tasa de AT varía de $36 \%$ a $93 \%$ en distintos estudios retrospectivos y prospectivos. Sus resultados sugieren que los médicos deberían privilegiar la asesoría a los pacientes sobre cómo mejorar su adherencia, en lugar de incrementar las dosis o el número de medicamentos.

En Latinoamérica, Quirós-Morales y VillalobosPérez (2007) estudiaron la AT de un grupo de 163 pacientes costarricenses con diabetes. Encontraron que los pacientes de zonas rurales mostraron mayor AT, probablemente por su mayor asistencia a pláticas educativas sobre la enfermedad. Por su parte, Riveros, Cortázar-Palapa, Alcázar y Sánchez-Sosa (2005), evaluaron los efectos de una intervención de tipo cognitivo conductual individual aplicada a una muestra de 51 pacientes con diabetes y/o hipertensión arterial. La intervención fue eficaz para mejorar las siguientes variables: calidad de vida, depresión, ansiedad, estilos de afrontamiento, bienestar y AT.

Para aumentar la AT frente a la DM2 es necesario implementar una intervención que ayude al paciente a explorar y resolver las ambivalencias acerca de su conducta o hábitos insanos y que pro- mueva cambios hacia estilos de vida más saludables. Actualmente, hay una intervención psicológica con dichas características denominada entrevista motivacional (EM) (Lizarraga \& Ayarra, 2009).

La EM ha mostrado su efectividad con pacientes con VIH/SIDA (Rollnick, Miller, \& Butler; 2008) y en pacientes con problemas de adicciones (Fuentes-Pila, Calatayud, López, \& Castañeda, 2005; Rollnick \& Miller, 1996; Sampl \& Kadden, 2004). La EM facilita que el paciente se posicione hacia el deseo de cambio y le ayuda a reconocer y ocuparse de sus problemas presentes y futuros, potenciando de esta forma su percepción de autoeficacia (Flórez-Alarcón \& Carranza, 2007). La EM aporta herramientas que permiten afrontar situaciones que no han podido ser resueltas mediante las estrategias habituales empleadas en promover cambios de conducta (Lizarraga \& Ayarra, 2009).

La EM está diseñada para fomentar la motivación intrínseca hacia el cambio en el paciente por medio de la resolución de su ambivalencia (Miller $\&$ Rollnick, 2002). En la EM se considera que la motivación y la percepción que tiene el sujeto de sus capacidades, entendidas como autoeficacia (Bandura, 1986), determinan parcialmente la conducta del individuo. Además, se considera que existen mecanismos psicobiológicos involucrados en la adquisición y mantenimiento de hábitos y estilos de vida. La EM es un método sistemático y colaborativo para ayudar a las personas a explorar sus propias motivaciones y valores, y a entender cómo estas pueden afectar sus niveles de adherencia. La EM enfatiza y respeta la autonomía del paciente, el cómo, cuándo o si desea cambiar, por lo tanto busca potenciar su motivación (Hettema, Steele, \& Miller; 2008).

Desde la perspectiva de la EM, es necesario que un paciente con DM2 tome en cuenta las consecuencias de su enfermedad y cómo le afectan en las distintas áreas de su vida, para que así logre iniciar un cambio que le permita alcanzar hábitos saludables (Morales \& García, 2010).

En 1992, Prochaska, DiClemente y Norcross mencionaron las fases del cambio por las cuales atraviesa una persona, las cuales son: (a) precontemplación, (b) contemplación, (c) preparación-ac- 
ción, (d) mantenimiento y (e) recaída. Estas fases $<$ forman parte fundamental de la EM. Miller y Rollnick (2002) enfatizaron que resulta indispensable que el terapeuta identifique la etapa del cambio en la que se encuentra el paciente, para así determinar el tipo de estrategia con la que va a intervenir. Por lo anterior, la EM promete ser una herramienta eficaz para incrementar el nivel de adherencia terapéutica de estos pacientes.

\section{Propósito del estudio}

Evaluar la eficacia de una intervención basada en la EM para incrementar la adherencia terapéutica de una muestra de pacientes mexicanos con DM2.

\section{Objetivos}

1. Identificar los factores que influyen en la adherencia terapéutica en pacientes mexicanos con DM2.

2. Identificar el nivel de eficacia de un programa de intervención basado en la EM cuyo objetivo es aumentar adherencia terapéutica de la muestra antes y después de la intervención.

\section{Método y materiales}

\section{Diseño}

Estudio cuasi experimental de dos grupos independientes, uno con intervención y otro sin intervención con evaluaciones pre-post.

\section{Muestra}

La muestra inicial estuvo conformada por 32 personas diagnosticadas con DM2 que acudían a consulta externa en el área de medicina interna en un hospital público del estado de San Luis Potosí (México), con un nivel de adherencia parcial o nulo de acuerdo con el MBG. La selección de los pacientes de la muestra general se hizo de manera no probabilística, por oportunidad. La asignación de los pacientes a los grupos la realizaron de manera aleatoria los médicos y la enfermera encargados del área a la que asistían los pacientes, con lo cual los autores del presente trabajo no participaron en este proceso. A los pacientes del grupo control se les ofreció la atención mediante la EM una vez concluida la intervención con el grupo experimental y una vez completada la evaluación post.

Criterios de inclusión: adultos, diagnóstico de DM2, nivel de AT parcial o nulo, aceptación voluntaria de participación. Criterios de exclusión: no adultos, no diagnóstico de DM2, nivel de AT óptimo, no aceptación voluntaria de participación.

\section{Procedimiento}

Se presentó el proyecto al Comité de Ética de la institución, mismo que previo dictamen aprobó su realización. Luego, los médicos y enfermeras del hospital invitaron a participar a los sujetos seleccionados; se pidió y obtuvo su consentimiento informado para la revisión de sus expedientes y para su participación en el estudio. Después de que los pacientes consintieron participar, se obtuvieron los datos sociodemográficos y los relativos a su enfermedad. Se realizó una entrevista semiestructurada -de la cual solamente se reportan algunos datos en el presente trabajo-, para conocer sus constructos personales en torno a su enfermedad; se revisaron sus expedientes para registrar los niveles de glucosa y se les administraron dos instrumentos, uno para medir el nivel de adherencia terapéutica y el otro para conocer su estilo de vida. Con base en el primer instrumento, se seleccionaron 22 pacientes que presentaron AT nula o parcial, mismos que conformaron la muestra final; de esta última, se seleccionaron de manera aleatoria un grupo control y un grupo experimental, el que contó con la intervención de entrevista motivacional, consistente en una o dos sesiones mensuales de 30 a 40 minutos (dependiendo de la disposición de los pacientes); posteriormente, se hizo una aplicación post de los instrumentos en ambos grupos.

La intervención consistió en un encuentro cara a cara de 30 a 40 minutos de duración. Aunque la intención original era que el plazo entre una consulta y otra fuera de 15 a 30 días, algunas pacientes cancelaron varias citas y, a pesar de que se logró 
reprogramarlas y alcanzar la meta de seis sesiones, las postergaciones y no iniciar la intervención simultáneamente con todos los pacientes obligaron a trabajar en esta fase durante ocho meses.

Las sesiones consistieron en ofrecer un espacio para que los pacientes hablaran acerca de las diferentes circunstancias de su vida y además se buscó que expresaran sus conductas ambivalentes en relación con su enfermedad, la percepción que tenían de sí mismos, de su familia, del servicio de salud, y se les motivaba de manera intrínseca al cambio hacia estilos de vida más saludables como hacer ejercicio, dieta, ingesta de medicamentos saludables y control emocional, entre otros, siempre con una actitud empática y validando sus fortalezas, habilidades y capacidades para el cambio.

Se procuró iniciar la intervención lo más cerca posible de los exámenes de glucosa de los pacientes, y se solicitó al personal médico que los prescribiera inmediatamente después de la intervención.

\section{Instrumentos}

- MBG: Cuestionario para Evaluar la Adherencia Terapéutica, diseñado y validado en Cuba por Martín, Bayarre y Grau (2004). La AT se clasifica como óptima (38 a 48 puntos), parcial (18 a 37 puntos) y no adherido (0 a 17 puntos). De acuerdo con los estudios de sus autores, la formulación de los ítems es razonable, se encuentran claramente definidos y se justifica su presencia en el cuestionario; presenta validez de contenido, índice de consistencia interna y un alfa de Cronbach de 0.889 . No se encontró un instrumento validado en México para evaluar la AT en DM2.

- Instrumento para Medir el Estilo de Vida en Pacientes con DM2 ([IMEVID]; López, Ariza, Rodríguez, \& Munguía, 2003). Posee validez lógica y de contenido, así como buen nivel de consistencia interna y externa con un valor de Cronbach de 0.81. Cuenta con estudios de validez en México, por ejemplo el de Resendiz-de-Leija, Felícitas-Ocampo Saldierna-Luque (2010), quienes concluyen que en el instrumento "existe asociación de estilo de vida y el dominio adherencia terapéutica con glucemia" (p. 22). Se eligió este instrumento por su validación en México y porque cuenta con una subescala que evalúa la AT, interés principal del estudio.

- Entrevista semiestructurada: fue diseñada expresamente para este estudio por Morales y García (2010). Consta de 25 preguntas abiertas; contiene preguntas sobre los datos sociodemográficos del paciente y sobre su enfermedad. Entre los ejes temáticos están: alimentación, medicamento, apoyo familiar y creencias en relación con la enfermedad, entre otros.

Se obtuvieron las medidas del nivel de glucosa que rutinariamente prescribían sus médicos (cada tres meses), mediante la revisión del expediente médico de los pacientes antes y después de la intervención, esto con la finalidad de evitar que les tomaran muestras adicionales de sangre, ya que los pinchazos fueron un motivo de molestia emocional y física cada vez que acudían a sus consultas. El personal de enfermería registró el peso y talla, siempre con el mismo equipo de medición en cada consulta; para esta investigación se consideraron los valores inmediatamente anteriores y posteriores a la intervención.

\section{Análisis estadístico}

El análisis estadístico se realizó utilizando el lenguaje estadístico R versión 2.9.2 (Ihaka \& Gentleman, 1996) con un $95 \%$ del nivel de confianza. Se estimaron datos faltantes utilizando el programa MICE (Multivariate Imputation by Chained Equations).

Se utilizó estadística no paramétrica para analizar correlaciones de los factores asociados a la DM2, ya que se trató de grupos pequeños de 11 participantes por grupo. Las mediciones se realizaron en escala ordinal, por lo cual se utilizó la correlación de Spearman. Asimismo, se empleó la prueba de Mann-Whitney para comparar los puntajes de los grupos control-experimental en las subescalas de 
los instrumentos IMEVID y MBG -estas pruebas están conformadas por reactivos de tipo Likert- y la prueba de Wilcoxon para comparar los puntajes pre-post de ambos grupos. Por otro lado, se usó la prueba $t$ de Student para realizar comparaciones del nivel de glucosa en los pacientes antes y después de la intervención. Por último, se llevó a cabo un análisis descriptivo individual de los puntajes de las pruebas de ambos grupos para identificar los cambios y/o diferencias observados antes y después de la intervención, y se interrogó a los sujetos del grupo experimental que no evidenciaron cambios en sus puntuaciones por las posibles razones.

\section{Resultados}

En ambos grupos, se observan más mujeres (ocho) que hombres (tres); la edad osciló entre 49 y 72 años para el grupo control y entre 47 y 72 años para el grupo experimental; predominó el estado civil casado en ambos grupos, variando la escolaridad entre primaria y licenciatura; para el grupo control, el promedio del Índice de Masa Corporal (IMC) fue de 28.39 y el promedio de glucosa fue de $170 \mathrm{mg} / \mathrm{dl}$; para el grupo experimental el promedio del IMC fue de 29.37 y el de glucosa de $157.91 \mathrm{mg} / \mathrm{dl}$; todos los sujetos se encontraban en un nivel de adherencia parcial, a excepción de un sujeto del grupo experimental, que se encontraba como No Adherido.

Primer objetivo: se encontró que los principales factores que influyen en la AT son; apoyo familiar $(r=0.784)$, creencias frente a la enfermedad $(r=$ $0.485)$, nivel de glucosa $(r=-0.4)$, disminución de la actividad sexual $(r=-0.447)$, reacciones emocionales frente a la enfermedad $(r=-0.44)$ y los años con DM2 $(r=-0.46)$; además se compararon si los años con diagnóstico influyen en el nivel de glucosa, 11 pacientes tenían menos de 10 años con el diagnóstico con el promedio de glucosa de $150.01 \mathrm{md} /$ $\mathrm{dl}$ y la otra mitad de pacientes con más de 10 años con el diagnóstico, con un promedio de glucosa de $168.54 \mathrm{md} / \mathrm{dl}$, encontrándose que dichas diferencias no fueron estadísticamente significativas,

Segundo objetivo: al comparar los resultados por escala y total del IMEVID en el pre entre el grupo control y el experimental, no se encontraron diferencias estadísticamente significativas, mientras que en el post se encontraron diferencias en

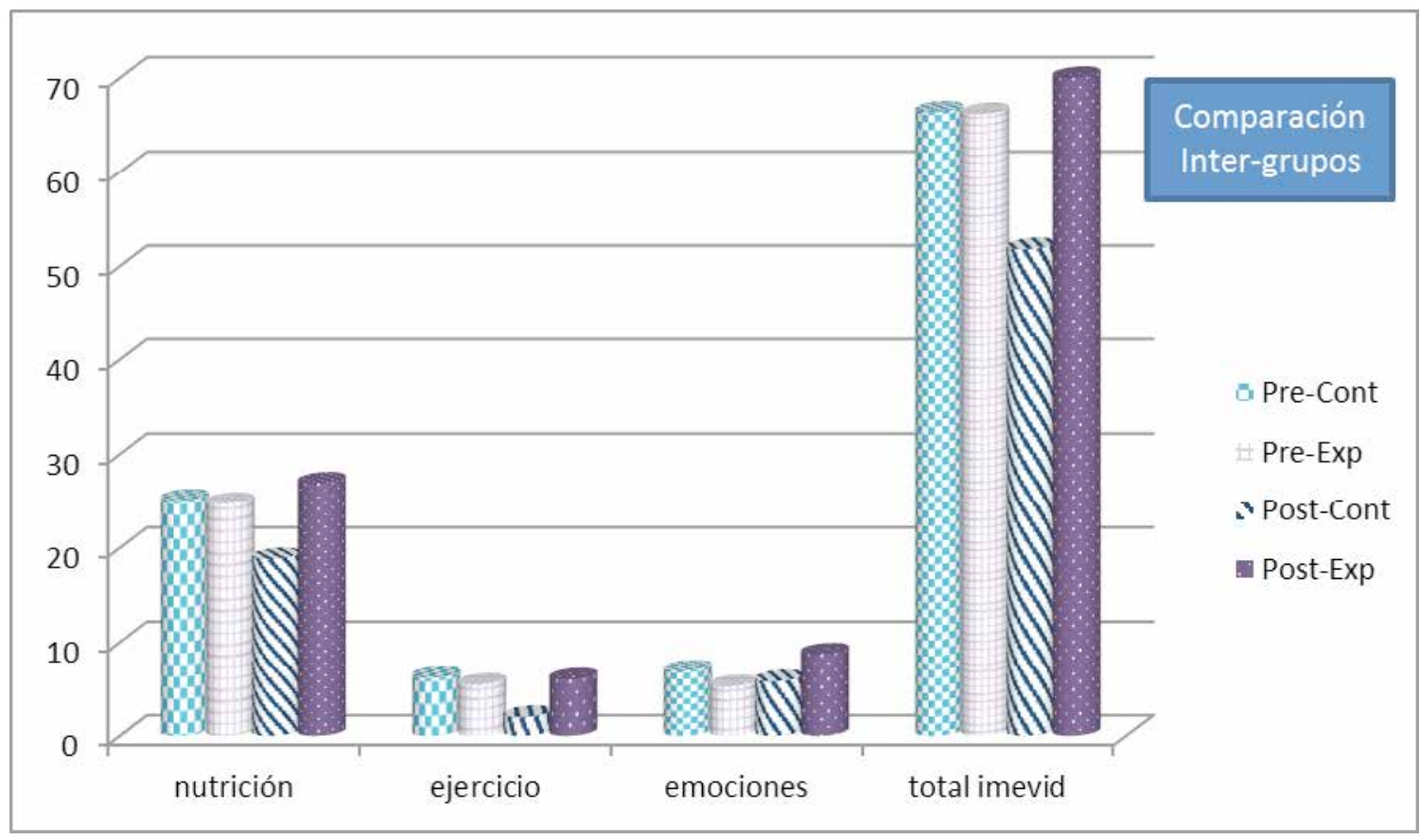

Grafica 1. Instrumento IMEVID. Dominios de estilos de vida predominantes en el grupo control y experimental en el pre y post.

Fuente: elaboración propia 
la subescala de nutrición $(z=-2.43 p \leq 0.01)$ y ejercicio $(z=-2.32 p \leq 0.02)$. Al comparar los resultados del pre contra el post de ambos grupos, se encontraron diferencias significativas en el grupo experimental en la subescala de emociones $(z=$ $-2.31 ; p \leq 0.02)$ y la escala total de adherencia $(z=$ $-2.64, p \leq 0.08)$. En ambos casos hubo una mejoría en el grupo experimental. En el grupo control solamente se encontraron diferencias significativas en la subescala de ejercicio $(z=-2.69, p \leq 0)$, aunque hubo diferencias casi significativas en la subescala de nutrición $(z=-1.91, p \leq 0.055)$ y adherencia total $(z=-1.73, p \leq 0.08)$. En estos últimos casos, los puntajes empeoraron. Se observó que el grupo experimental tiende consistentemente a aumentar hacia conductas de estilos de vida más saludables, mientras que en el grupo control disminuyeron. En la Gráfica 1 se muestran los resultados del pre y del post del grupo control y experimental del IMEVID.

Como parte del segundo objetivo, se realizó la comparación estadística de los valores del MBG antes y después de la intervención, y se encontraron diferencias significativas en el grupo experimental $(z=-2.18, p \leq 0.02)$. Como se muestra en la Grá- fica 2, en el grupo control se observa que todos los sujetos tenían adherencia parcial y en su post evaluación solamente hubo un cambio: un sujeto cambio su nivel de AT a total. En el grupo experimental, en el pre 10 sujetos estaban en el nivel de adherencia parcial y un sujeto como no adherido; en el post, seis sujetos cambiaron su nivel parcial a adherencia óptima, uno pasó de adherencia nula a parcial y los cuatro restantes continuaron con el nivel parcial.

Para concluir el análisis estadístico, se hizo una comparación entre los niveles de glucosa de los pacientes antes y después de la intervención. La comparación estadística de dichos niveles de glucosa entre el pre y el post del grupo experimental mostró diferencias significativas, al analizar los datos con la $t$ de Student $(t=-2.068, p \leq 0.05)$; lo que no sucedió con el grupo control (en el cual, la adherencia incluso empeoró).

En la Tabla 1 se pueden apreciar los valores de los niveles de glucosa de los pacientes del grupo control y del experimental antes y después de la intervención. Como se puede ver, solamente tres sujetos del grupo control disminuyeron sus niveles

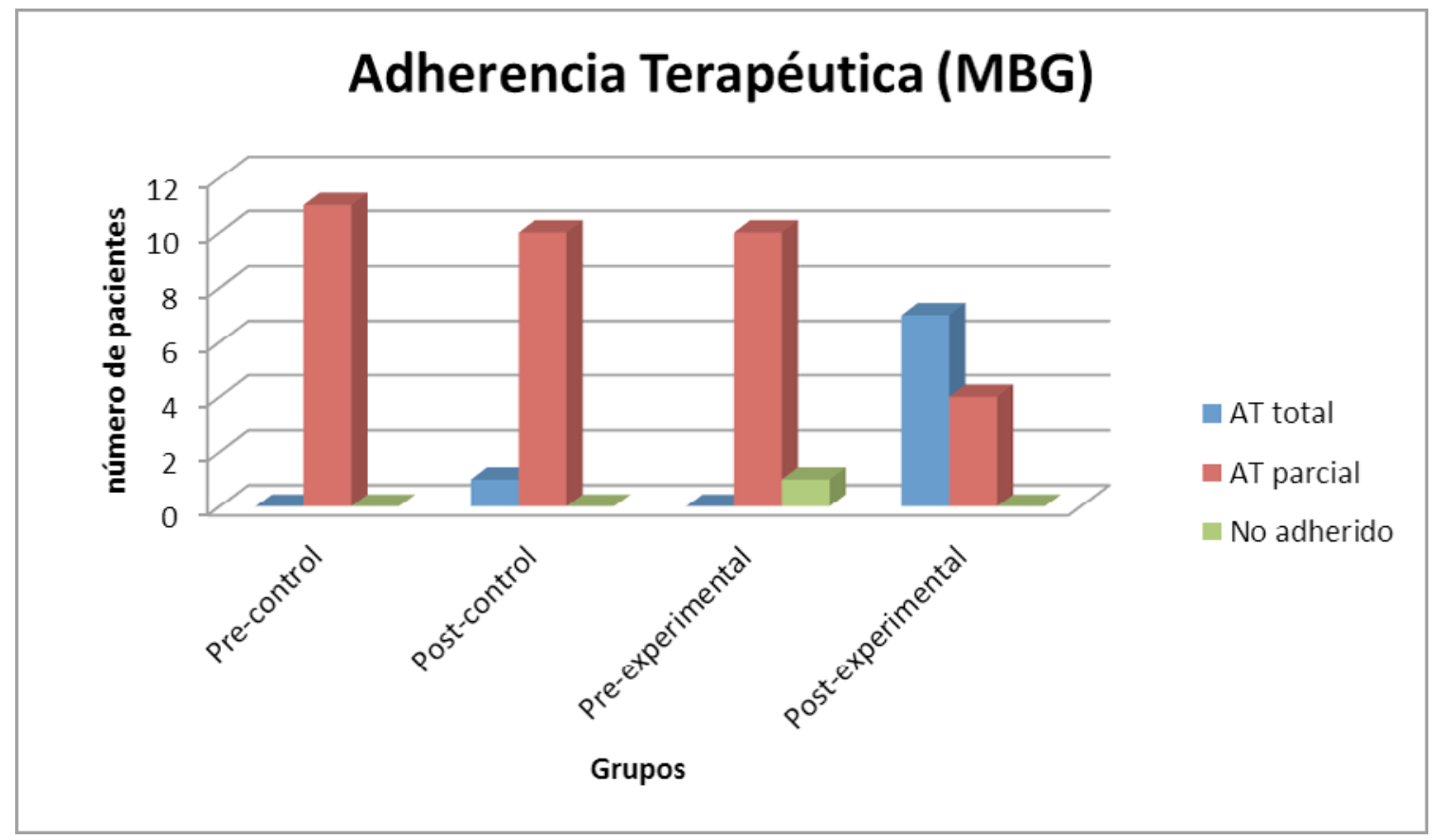

Gráfica 2. Nivel de Adherencia Terapéutica antes y después de la intervención (Instrumento MBG).

Fuente: elaboración propia 
TABLA 1

Nivel de glucosa pre-post del grupo control y del grupo experimental

\begin{tabular}{cccccc}
\hline \multicolumn{6}{c}{ Nivel de glucosa } \\
\hline Gujeto control & Pre & Post & sujeto & Gpo experimental \\
\hline 1 & 148 & 233 & 1 & 150 & Post \\
2 & 160 & 140 & 2 & 71 & 90 \\
3 & 198 & 220 & 3 & 220 & 110 \\
4 & 144 & 109 & 4 & 276 & 120 \\
5 & 226 & 255 & 5 & 241 & 230 \\
6 & 153 & 233 & 6 & 198 & 109 \\
7 & 181 & 180 & 7 & 99 & 106 \\
8 & 131 & 145 & 8 & 80 & 65 \\
9 & 142 & 190 & 9 & 129 & 142 \\
10 & 238 & 145 & 10 & 124 & 105 \\
11 & 150 & 156 & 11 & 149 & 233 \\
\hline $\mathrm{X}$ & 170.09 & 182.36 & $\mathrm{X}$ & 157.91 & 136.36 \\
\hline
\end{tabular}

Fuente: elaboración propia

de glucosa (no se considera a un paciente que disminuyó un solo punto este indicador). En promedio, aumentaron doce puntos en sus niveles de glucosa entre ambas evaluaciones. Por su parte, seis sujetos del grupo experimental disminuyeron sus niveles de glucosa. Como grupo, disminuyeron 21 puntos el mencionado nivel.

\section{Discusión}

En los datos sociodemográficos de ambos grupos se encontró que predomina el género femenino. Esto es importante porque el afrontamiento de la enfermedad para las mujeres es distinto que para los hombres, debido a que presentan mayor tensión, ansiedad y depresión; asimismo, cuentan con mayor masa corporal, escasa oportunidad de diversión activa, función múltiple de roles y atención escasa de su enfermedad, entre otros (Salcedo-Rocha, García de Alba-García, Frayre-Torres, \& LópezCoutino, 2008).

El promedio de edad para los dos grupos fue de 58 años, predominando el estado civil casado, y la escolaridad variando desde primaria hasta licenciatura. El IMC fue, en promedio, de 28.39 para el grupo control y de 29.37 para el grupo experimental; en ambos grupos se observó sobrepeso y obesidad, factor importante y de alto riesgo en la prevalencia de la DM2 (INSP, 2013).

Entre los factores relacionados con la AT del presente estudio, se encontró el apoyo familiar (especialmente de los hijos). El paciente diabético está en la cuarta etapa del ciclo vital familiar (recordando que la media de edad de los pacientes fue de 58 años), que se inicia con la primera salida del hijo del hogar familiar (Fernández, 2004) y emerge entonces la necesidad del apoyo de los hijos para seguir adelante con las prescripciones de su tratamiento, pues cuando un miembro de la familia padece alguna enfermedad crónica, necesita el apoyo de algún miembro de la familia para el seguimiento de sus consultas, para llevar una dieta adecuada, para el cumplimiento de la ingesta farmacológica, etc.; las pacientes del presente estudio reportaron que las cancelaciones de las consultas se debieron a que no había quién las transportara y acompañara a la institución de salud, y que el sostén familiar tanto económico como moral para asistirlas eran sus hijos. Por otro lado, los miembros de la familia también necesitan educación, al igual que el paciente, para ayudar a lograr los cambios necesarios en el estilo de vida (Cifuentes \& Quintul, 2005). El apoyo familiar para que el paciente llevara a cabo alguna actividad física también se encontró favorable en el estudio. 
Otro factor relacionado con la AT fue el de creencias frente a la enfermedad. Los pacientes reportaron adjudicar el origen de la DM2 a situaciones de estrés que han presentado durante su vida, relacionado con pérdidas, impresiones fuertes, complicaciones de salud, accidentes y problemas laborales. De acuerdo con la literatura, el origen del estrés puede ser físico o mental: cuando aparece, el cuerpo se prepara para actuar y segrega niveles de distintos tipos de hormonas con el objetivo de proporcionar a las células una gran cantidad de la energía almacenada (glucosa y grasa); en las personas con DM2 la insulina no siempre es capaz de trasladar la energía extra a las células y por consiguiente la glucosa se acumula en la sangre, provocando un alto grado de glucemia (American Diabetes Association, 2011).

Los resultados mostraron que hubo algunas diferencias estadísticamente significativas entre los puntajes de las escalas de nutrición y ejercicio del IMEVID, al comparar los resultados del post del grupo control y del experimental. Al comparar los puntajes entre el pre y el post en ambos grupos, solamente se encontraron diferencias significativas en la subescala de emociones y total del grupo experimental, y de ejercicio, en el control.

Los pacientes del grupo experimental que lograron cambios significativos en la escala total del IMEVID reportaron que a partir de la intervención empezaron a comer de manera más saludable, iniciando con la disminución paulatina del consumo de azúcares, grasas y harinas, dándose 'permiso' para ingerir alimentos no permitidos de manera muy ocasional e incrementando frutas, verduras y bebidas naturales; asimismo, reportaron estrategias de alimentación más apropiadas en eventos o festejos, como comer en casa antes de asistir al evento o bien llevar alimentos saludables. En cuanto al ejercicio, empezaron con actividad física leve, como estiramientos, caminatas y en algunos casos baile y aeróbicos. Por último, en el área emocional, los pacientes mencionaron contar con nuevas perspectivas de su futuro, empezaron a planear viajes, mostraron interés por conocer a sus nietos, iniciar nuevas relaciones de pareja (los pacientes viudos); además, reportaron que, al sentirse con más ánimo y optimismo, empezaron a buscar ocupaciones donde ejercieran un oficio, como vendedoras, empleadas de supermercados, negocios propios, entre otros. En contraste, los pacientes del grupo control disminuyeron de manera consistente sus puntajes en todas las escalas del IMEVID, incluyendo la escala total.

Por otro lado, se encontraron diferencias significativas en los puntajes totales del MBG, mostrando una mayor adherencia terapéutica en el post del grupo experimental. Los sujetos del grupo experimental reportaron tomar sus medicamentos en las dosis y horarios establecidos, asumiendo una mayor responsabilidad y participación en su tratamiento. Por su parte, los pacientes del grupo control disminuyeron sus puntajes en este instrumento, aunque dicha disminución no fue significativa.

La Federación Mexicana de Diabetes (FDM, 2010) considera como normales los niveles de glucosa entre 70 y $90 \mathrm{mg} / \mathrm{dl}$. Una cifra menor a 70 es considerada como hipoglucemia (niveles muy bajos de glucosa en sangre) y los niveles por encima de $126 \mathrm{mg} / \mathrm{dl}$ han demostrado ser perjudiciales para la salud. En el presente estudio, se encontró que en el pre para ambos grupos, la glucemia se encontró en un rango arriba de lo normal $(170.09 \mathrm{mg} / \mathrm{dl}$ en grupo control y $157.91 \mathrm{mg} / \mathrm{dl}$ en grupo experimental); en el post, la glucemia del grupo control fue de $182.36 \mathrm{mg} / \mathrm{dl}$ y del experimental $136.36 \mathrm{mg} / \mathrm{dl}$; como es lógico, se encontró que a mayor nivel de glucosa, menor nivel de adherencia terapéutica. También se encontró que a mayor nivel de glucosa corresponde una disminución de la actividad sexual (lo cual coincide con lo mencionado por Romero $\&$ Licea, 2000) y pobre o escaso control de reacciones emocionales negativas sobre su enfermedad (Arceaga-Domínguez, Lara Muñoz \& Ponce de León-Rosales, 2005).

El promedio de glucosa para los seis sujetos del grupo experimental que lograron cambios de nivel de adherencia parcial a total fue de $123 \mathrm{mg} / \mathrm{dl} \mathrm{y}$ la de los cuatro pacientes que continuaron en el mismo nivel de AT (parcial) fue de $163 \mathrm{mg} / \mathrm{d}$, encontrándose que la razón por la cual estos pacientes continuaron en el mismo nivel de adherencia fue probablemente su asistencia irregular a las sesiones de intervención psicológica (los pacientes reporta- 
ron que no podían trasladarse por sí mismos y que no contaron con el apoyo para hacerlo, lo que obligó a reprogramar sus sesiones).

$\mathrm{Al}$ analizar los resultados del $\mathrm{MBG}$ y los niveles de glucosa se encontró que tres de las pacientes que no variaron sus puntajes en el MBG eran mujeres, las cuales tampoco disminuyeron sus niveles de glucosa (dos pacientes mujeres sí cambiaron sus puntaje del MBG, pero no los de glucosa). Lo anterior es muy importante porque muestra que se debe utilizar una perspectiva de género al diseñar una intervención, pues probablemente por razones personales y culturales, las mujeres enfrentan retos mayores para mejorar su AT.

Los pacientes del grupo experimental reportaron que durante la intervención se sintieron comprendidos, pero sobre todo que les benefició que alguien los tomara en cuenta como seres humanos; comentaron que compartir las dificultades que experimentan en el transcurso de su enfermedad les servía para adoptar un estilo de vida más saludable; mencionaron que les gustaría que se implementara un servicio donde contaran con la posibilidad de acceder a un profesional para platicar sobre su enfermedad y que los apoyara en sus recaídas con amabilidad, respeto y comprensión.

El presente estudio tiene varias limitaciones: no hubo un control estricto en cuanto al registro de los niveles de glucosa y el inicio del tratamiento, no se pudo realizar la intervención en un tiempo más acotado, se utilizó un instrumento validado en México, pero que no evaluaba de manera más directa la AT y se trabajó con muy pocos sujetos. Se sugiere tomar en cuenta estas limitaciones al momento de considerar los resultados de la presente investigación.

\section{Conclusiones}

Aun cuando no hubo diferencias significativas en todas las comparaciones estadísticas, en la mayoría de los pacientes del grupo experimental se observó mejoría en cuanto a la adherencia terapéutica después de la intervención y en su comparación con el grupo control. Esto se observó en el IMEVID. El puntaje total para el grupo control en el post fue de 51.8 y el del grupo experimental fue de 70, favoreciéndose este último grupo, el cual aumentó las conductas de estilos de vida saludables deseables en los pacientes. Por otra parte, en el MBG hubo diferencias significativas en la comparación pre-post del grupo experimental. Lo anterior se refleja en el hecho de que solo una persona del grupo control cambio de nivel de AT de parcial a total y en el grupo experimental seis personas cambiaron de adherencia parcial a adherencia total y un sujeto de no adherido a adherencia parcial. Por otro lado, en cuanto al nivel de glucosa post para el grupo control fue $182 \mathrm{mg} / \mathrm{dl}$ y para el experimental fue de 136. Los resultados anteriores nos permiten concluir que la intervención psicológica basada en la entrevista motivacional fue eficaz para aumentar el nivel de adherencia terapéutica de un grupo de pacientes con DM2, los cuales eran atendidos en una institución pública de salud. La falta de intervención se reflejó en resultados más pobres en el grupo control.

En el presente estudio, se resalta la importancia de fomentar una intervención psicológica que apoye a los pacientes para que incrementen o mantengan su AT, no solo para aumentar su motivación, sino para lograr cambios conductuales efectivos. Se sugiere la entrevista motivacional como estrategia de apoyo a los pacientes, pues fomenta una relación de respeto, de apoyo y de colaboración entre el proveedor de servicio (en este caso el psicólogo) y los pacientes, relación muy distinta a la que habitualmente establecen los pacientes con los proveedores de servicios de salud. Apoyar a los pacientes a mejorar o mantener su AT beneficia no solamente al sistema de salud, por los costos tan altos asociados a una mala AT, sino que beneficia a los pacientes, pues les permite alcanzar o mantener la calidad de vida a la que tienen derecho.

\section{Referencias}

American Diabetes Association (2013). Lo básico sobre la diabetes tipo 2. Recuperado de http://www. diabetes.org/es/informacion-basica-de-la-diabetes/ diabetes-tipo-2/lo-basico-sobre-la-diabetes-tipo2. html 
Arceaga-Domínguez, A., Lara Muñoz, C., \& Ponce de León-Rosales, S. (2005). Revista de Investigación Clínica, 57(5), 676-684.

Bandura, A. (1986). The explanatory and predictive scope of self-efficacy theory. Journal of Clinical and Social Psychology, 4, 359-373.

Balkrishnan, R., Rajagopalan, R., Camacho, F., Huston, S., Murray, F., \& Anderson, R. (2003). Predictors of medication adherence and associated health care costs in an older population with type 2 diabetes mellitus: A longitudinal cohort study. Clinical Therapeutics, 25(11), 2958-2971.

Cifuentes, J., \& Quintul, N. (2005). La familia: iapoyo o desaliento para el paciente diabético? Programa de Diplomado en Salud Pública y Salud Familiar. Recuperado de http://medicina.uach.cl/saludpublica/ diplomado/contenido/trabajos/1/Osorno\%202005/ La_Familia_y_el_paciente_diabetico.pdf

Cramer, J. (2004). A systematic review of adherence with medications for diabetes. Diabetes Care, 27(5), 1218-1224.

DiMatteo, R., Lepper, H., \& Croghan, T. (2000). Depression is a risk factor for noncompliance with medical treatment. Archives of Internal Medicine, 160, 2102-2107.

Durán, B., Rivera, B., \& Franco, E. (2001). Apego al tratamiento farmacológico en pacientes con diagnóstico de diabetes mellitus tipo 2. Revista de Salud Pública, 43(3), 233-236.

Federación Mexicana de Diabetes. (2010). Disponible en http://www.fmdiabetes.org/fmd/pag/factores. php?id=MTAx

Fernández, M. A. (2004). El impacto de la enfermedad en la familia. Revista de la Facultad de Medicina, 47(6), 1-5.

Flórez-Alarcón, L., \& Carranza, W. (2007). La entrevista motivacional como herramienta para el fomento de cambios en el estilo de vida de personas con enfermedades crónicas no transmisibles. Avances en Psicología Latinoamericana, 25(2), 63-82.

Fuentes-Pila, J. M., Calatayud, P., López, E., \& Castañeda, E. (2005). La entrevista motivacional: llave del proceso de cambio en la dependencia nicotintabáquica. Trastornos Adictivos, 7(3), 153-165.

Hernández-Ávila, M., Gutiérrez, J. P., \& ReynosoNoverón, N. (2013). Diabetes mellitus en México.
El estado de la epidemia. Salud Pública de México, 55(Supl. 2), 29-S136.

Hernández-Romieu, A., Elnecavé-Olaiz, A., HuertaUribe, N., \& Reynoso-Noverón, N. (2011). Análisis de una encuesta poblacional para determinar los factores asociados al control de la diabetes mellitus en México. Salud Pública de México, 53(1), 34-39.

Hettema, J., Steele, J., \& Miller, W. (2008). Entrevista motivacional. Revista de Toxicomanía, 52, 3-24.

Ihaka, R., \& Gentleman, R. (1996). R: A language for data analysis and graphics. Journal of Computational and Graphical Statistics, 5, 299-314.

International Diabetes Federation. (2013). Atlas de diabetes de la FID (5.- ed.). Bruselas: Autor. Recuperado de http:// http://www.idf.org/diabetesatlas/5e/ es/prologo

Leytur, O. (2002). El paciente diabético. Recuperado de http://fbio.uh.cu/bqesp/nutricion/diabetico

Lizarraga, S., \& Ayarra, M. (2009). Entrevista motivacional. Anales del Sistema Sanitario de Navarra, 24(Supl. 2), 43-53.

López, J., Ariza, C., Rodríguez, J., \& Munguía, C. (2003). Construcción y validación inicial de un instrumento para medir el estilo de vida en pacientes con diabetes mellitus tipo 2. Salud Pública de México, 45(4), 259-268.

Martín, L., Bayarre, H., \& Grau, J. (2004). La investigación de la adherencia terapéutica como un problema de la psicología de la salud.. Revista Psicología y Salud, 14(1). Recuperado de http://www.uv.mx/ psicysalud/psicysalud_14_1/14_1/

LMart\%C3\%ADn.htmlarttext\&pid=S086434662008 $000100012 \& \operatorname{lng}=\mathrm{es}$

Mena, F. J., Martín, J. C., Simal, F., Bellido, J., \& Carretero, J. L. (2006). Diabetes mellitus tipo 2 y calidad de vida relacionada con la salud: resultados del Estudio Hortega. Anales de Medicina Interna, 23(8), 357-360.

México, Gobierno de la República. (2013). Estrategia nacional para la prevención y el control del sobrepeso, la obesidad y la diabetes. México: Autor. Recuperado de 2014 http://promocion.salud.gob.mx/dgps/descargas1/estrategia/Estrategia_con_portada.pdf

México, Instituto Nacional de Estadística y Geografía. (2013). Estadísticas a propósito del día mundial de la diabetes. Recuperado de http://www.inegi.org. 
$\mathrm{mx} /$ inegi/contenidos/espanol/prensa/contenidos/ estadisticas/2013/diabetes0.pdf

México, Instituto Nacional de Salud Pública. (2012). Encuesta nacional de salud y nutrición 2012. México: Autor.

México, Instituto Nacional de Salud Pública. (2013). Encuesta nacional de salud y nutrición 2012. Resultados por entidad federativa. San Luis Potosí. México: Autor.

México, Secretaría de Salud. (2007). Programa nacional de salud 2007-2012. México: Autor.

México, Secretaría de Salud. (2013). Programa sectorial de salud. México: Autor.

Miller, W., \& Rollnick, S. (2002). Motivational interviewing: Preparing people for change (2. $\cdot$ ed.). New York: Guilford Press.

Miller, W., \& Rollnick, S. (2004). Talking oneself into change. Journal of Cognitive Psychotherapy, 18(4), 299-308.

Morales, B. V., \& García, I. (mayo, 2010). Eficacia de la entrevista motivacional para promover la adherencia terapéutica en pacientes con DM2. Trabajo presentado en el XXXVII Congreso del Consejo Nacional para la Enseñanza e Investigación en Psicología, Chihuahua, México.

Organización Mundial de la Salud. (noviembre, 2012). Diabetes (Nota descriptiva N. -312 ). Consultado en http://www.who.int/mediacentre/factsheets/fs312/es/

Organización Panamericana de la Salud. (2011). Recuperado de http://new.paho.org/hq/

Prochaska, J., DiClemente, C., \& Norcross, J. (1992). In search of how people change. Applications to addictive behaviors. American Psychologist, 47(9), 1102-1114.

Quirós-Morales, D., \& Villalobos-Pérez, A. (2007). Comparación de factores vinculados a la adherencia al tratamiento en Diabetes Mellitus tipo ii entre una muestra urbana y otra rural de Costa Rica. Universitas Psychologica, 6(3), 679-688.

Resendiz-de-Leija, R., Felícitas-Ocampo, A., \& Saldierna-Luque, A. L. (2010). Asociación de los do- minios (estilo de vida) de la encuesta IMEVID, con glucemia, en pacientes con diabetes tipo 2. Revista de Sanidad Militar, 64(5), 211-223.

Riveros, A., Cortázar-Palapa, J., Alcázar, F., \& SánchezSosa, J. J. (2005). Efectos de una intervención cognitivo-conductual en la calidad de vida, ansiedad, depresión y condición médica de pacientes diabéticos e hipertensos esenciales. International Journal of Clinical and Health Psychology, 5(3), 445-462.

Rodríguez Bolaños, R. A., Reynales Shigematsu, L. M., Jiménez Ruíz, J. A., Juárez Márquez, S. A., \& Hernández Ávila, M. (2010). Costos directos de atención médica en pacientes con Diabetes Mellitus tipo 2 en México: análisis de microcosteo. Revista Panamericana de Salud Pública, 28(6), 412-420.

Romero, J. C., \& Licea, M. E. (2000). Disfunción sexual eréctil en la diabetes mellitus. Revista Cubana de Endocrinología, 11(2), 105-20.

Rollnick, S., Miller, W., \& Butler, C. (2008). Motivational interviewing in health care: Helping patients change behavior. New York: The Guilford Press.

Rollnick, S., \& Miller, W. (1996). ¿Qué es la entrevista motivaciónal? Revista de Toxicomanías, 6, 3-7.

Salcedo-Rocha, A., García de Alba-García, J., FrayreTorres, M., \& López-Coutino, B. (2008). Género y control de diabetes mellitus 2 en pacientes del primer nivel de atención. Revista Médica del Instituto Mexicano del Seguro Social, 46(1), 73-81.

Sampl, S., \& Kadden, R. (2004). Terapia de estimulación motivacional cognitivo-conductual para adolescentes adictos a la marihuana. Barcelona: Ars. Medica.

Vermeire, E. I. J. J., Wens, J., Van Royen, P., Biot, Y., Hearnshaw, H., \& Lindenmeyer, A. (2005). Interventions for improving adherence to treatment recommendations in people with type 2 diabetes mellitus. Cochrane Datbase of Systematic Reviews, 2005(2), Art. CD003638. doi: 10.1002/14651858. cd003638.pub2 